\title{
Implantação de uma Comissão de Cuidados com a Pele em um Hospital de Ensino*
}

Implementation of a Skin Care Commission in a University Hospital

\author{
Implementación de una Comisión de Cuidado de la Piel en un Hospital Universitario
}

Tereza Cristina Caron Krause?, Gisela Maria Assis², Mitzy Tannia Reichembach Danski³

\begin{abstract}
RESUMO
Objetivo: Foi descrever as fases de implantação de uma Comissão de Cuidados com a Pele em um hospital de ensino. Método: Pesquisa descritiva documental, realizada no período de fevereiro de 2012 a março de 2014, por meio de consulta a documentos institucionais. Resultados: A implantação foi descrita nas seguintes fases por fins didáticos: negociação - apresentação da proposta à Diretoria de Enfermagem, Direção Geral e seus respectivos colegiados; estruturação - formação do núcleo estruturador, com reuniões periódicas para deliberações quanto aos membros e ao funcionamento da Comissão; documentação - elaboração de projeto, regimento, ofícios, solicitações de padronização, instrumentos de avaliação, relatórios, entre outros; implementação - funcionamento de reuniões, capacitações, avaliações e acompanhamento dos pacientes. Conclusão: Considera-se efetiva a estratégia utilizada para implantação da Comissão de Cuidados com a Pele e sugere-se a participação multiprofissional na sua composição. Destaca-se a importância da criação de comissões para a implementação do plano de segurança do paciente.
\end{abstract}

DESCRITORES: Estomaterapia. Comitê de Profissionais. Ferimentos e lesões. Segurança do paciente.

\begin{abstract}
Objective: It was to describe the implementation phases of a Commission for Skin Care in a university hospital. Method: Documentary, descriptive study conducted from February 2012 to March 2014, through consultation with institutional documents. Results: The implantation was described in the following phases for teaching purposes: negotiation - presentation of the proposal to the Board of Nursing, General Direction and their respective collegiate; structuring - formation of structuring core with regular meetings for deliberations as Members and functioning of the Commission; documentation - project design, regiment, crafts, requests for standardization, assessment tools, reports, etc.; implementation - running meetings, training, evaluations and monitoring of patients. Conclusion: It is considered effective the strategy used to implement the Skin Care Commission and it is suggest the multidisciplinary participation in its composition. Highlights on the importance of creating committees for the implementation of the security plan of the patient.
\end{abstract}

DESCRIPTORS: Stomatherapy. Professional Staff Committees. Wounds and injuries. Patient safety.

\footnotetext{
'Enfermeira, Mestre em Enfermagem pelo Programa de Pós-Graduação Mestrado Profissional em Enfermagem da Universidade Federal do Paraná (UFPR); Membro do Núcleo Estruturador da Comissão de Cuidados com a Pele do Hospital de Clínicas de Curitiba da UFPR - Curitiba (PR), Brasil Endereço para correspondência: Rua Herculano Carlos Franco de Souza, 550, apto 604 A - CEP: 80240-290 - Curitiba (PR), Brasil - E-mail: terezakrause@onda.com.br Enfermeira; Mestre em Tecnologia em Saúde pelo Programa de Pós-Graduação em Enfermagem da Pontifícia Universidade Católica (PUC) do Paraná; Coordenadora da Comissão de Cuidados com a Pele do Hospital de Clínicas de Curitiba da UFPR - Curitiba (PR), Brasil.

${ }^{3}$ Enfermeira, Doutora em História; Professora Adjunta da Graduação e Programa de Pós-Graduação do departamento de Enfermagem da UFPR Curitiba (PR), Brasil.

*Extraído da dissertação "Implantação de uma Comissão de Cuidados com a Pele em Hospital de Ensino". Programa de Pós-Graduação em Enfermagem Mestrado Profissional da Universidade Federal do Paraná em 2013.

Artigo recebido em: 01/04/2015 - Aceito para publicação em: 22/04/2015
} 


\section{RESUMEN}

Objetivo: Fue describir las fases de la implementatión de una Comisión para Cuidado de la piel en un hospital universitario. Método: Documental, estudio descriptivo realizado entre febrero 2012 y marzo 2014 , a través de consultas con los documentos institucionales. Resultados: La implantación se describe en las siguientes fases con fines didácticos: negociación - presentación de la propuesta a la Dirección de Enfermería, Dirección General y sus respectivos colegiados; Estructuración - formación de núcleo de estructuración con reuniones periódicas para las deliberaciones de miembros y funcionamiento de la Comisión; documentación - diseño de proyectos, regimiento, la artesanía, las solicitudes de normalización, herramientas de evaluación, informes, etc.; implementación - ejecución de reuniones, formación, evaluación y seguimiento de los pacientes. Conclusión: Se considera efectiva la estrategia utilizada para implementar la Comisión y se sugieren la participación multidisciplinaria en su composición. Se destaca la importancia de la creación de comités para la ejecución del plan de seguridad del paciente.

DESCRIPTORES: Estomaterapia. Comités de Profesionales. Heridas y traumatismos. Seguridad del paciente.

\section{INTRODUÇÃO}

A segurança do paciente é um assunto de interesse internacional, considerado pela Organização Mundial da Saúde um princípio fundamental do cuidado à saúde, pois no processo de cuidar existe certo grau de insegurança. Cuidados de saúde inseguros geram uma exposição maior a riscos resultando em acréscimo da morbidade e mortalidade evitáveis e em gastos adicionais com a manutenção dos sistemas de saúde ${ }^{1}$. As exigências de segurança e de controle nos serviços de saúde crescem com a evolução tecnológica, despertando cada vez mais nos profissionais de saúde a preocupação com a segurança dos pacientes ${ }^{2}$.

Dessa forma, evidencia-se a necessidade do uso de indicadores de qualidade em consonância com a política de segurança nacional, impactando na qualidade da assistência de Enfermagem ${ }^{3}$.

Ressalta-se a necessidade de evitar que a assistência prestada resulte em dano ao paciente. Para tanto, deve-se trabalhar na prevenção dos eventos adversos, que ocorrem quando o cuidado é prestado sem seguir os padrões estabelecidos para a prevenção e o tratamento de uma condição específica, como a úlcera por pressão, lesão por umidade, lesão por adesivo, lesão por fricção, entre outros ${ }^{4}$.

As lesões de pele constituem um problema de saúde, com graves consequências na qualidade de vida dos pacientes, no tempo da assistência de Enfermagem e na utilização e custos dos recursos materiais, sendo um grande desafio para os enfermeiros ${ }^{5}$.

O tratamento do paciente com ferida deve ser dinâmico e precisa acompanhar a evolução científica e tecnológica, visando à avaliação do paciente, indicação do tratamento, organização e sistematização da assistência. Portanto, faz-se necessário para o profissional de Enfermagem que trabalha com prevenção e tratamento de feridas o conhecimento da fisiologia da pele, da cicatrização, dos fatores de risco e das etapas do processo, como também dos produtos disponíveis no mercado e sua correta indicação ${ }^{6}$.

Diariamente, em um hospital de ensino de grande porte de Curitiba, diversos pacientes com feridas complexas ou alto risco para lesões de pele são atendidos ou internados, entre eles pacientes com insuficiências vasculares, diabetes, lesão medular, neoplasias e outras enfermidades. Ambulatorialmente, a demanda é atendida pelo ambulatório de tratamento de feridas, porém não existe nenhuma forma de assistência sistematizada aos pacientes internados no que diz respeito à prevenção ou ao tratamento de feridas e outras alterações de pele, tampouco encaminhamento desses pacientes para continuidade da assistência necessária.

Com esta publicação, que objetivou descrever os passos de implantação de uma Comissão de Cuidados com a Pele, pretende-se auxiliar profissionais da saúde no caminho para soluções similares, para que cada vez menos pacientes estejam expostos aos riscos de uma assistência não sistematizada.

\section{MÉTODO}

Trata-se de uma pesquisa descritiva documental, realizada em um hospital de ensino de grande porte, de média e alta complexidade, com 510 leitos distribuídos em 25 unidades 
de internação nas áreas clínica, cirúrgica, pediátrica e maternidade, totalizando 59 especialidades, na cidade de $\mathrm{Curitiba}^{7}$.

A coleta de dados foi realizada no período de fevereiro de 2012 a março de 2014 por meio de consulta autorizada a documentos institucionais relacionados produzidos nesse período.

Foram utilizados como fonte: atas do grupo de trabalho da Comissão de Feridas (anterior à alteração de nome da Comissão), do núcleo estruturador da Comissão, de reuniões gerais da Comissão, dos subgrupos de feridas crônicas, feridas agudas, estomias e cateteres e drenos, atas de reuniões ordinárias do Colegiado Assessor Técnico da Diretoria de Enfermagem, colegiado gestor, pré-projeto e projeto de implantação da Comissão, regimento interno, portaria de nomeação dos membros, ofícios internos, fluxogramas, procedimentos operacionais padrão descritos, instrumentos de avaliação e acompanhamento de pacientes, relatório de atividades e fichas de solicitação de padronização de materiais.

$\mathrm{O}$ projeto de pesquisa foi aprovado pelo Comitê de Ética em Pesquisa em Seres Humanos do Setor de Ciências da Saúde da Universidade Federal do Paraná, sob o registro No CONEP/CAAE: 07555012.8.0000.0102.

\section{RESULTADOS}

A implantação da Comissão encontra-se descrita em quatro fases - negociação, estruturação, documentação e implementação - , para melhor compreensão, porém vale salientar que em diversos momentos mais de uma fase esteve em andamento concomitantemente.

\section{Negociação}

A ideia de implantação da Comissão nasceu em orientação de mestrado profissional, no grupo de pesquisa de Tecnologia e Inovação em Saúde, no qual uma das autoras estava inserida como mestranda. A proposta inicial era de implantação de um Comitê de Prevenção e Tratamento de Úlceras por Pressão, aprovada por unanimidade na reunião do Colegiado Assessor Técnico da Diretoria de Enfermagem do hospital de ensino deste estudo em 6 de dezembro de 2011.

A proposta da Comissão inicial foi ampliada para Comissão de Prevenção e Tratamento de Feridas, por sugestão da Diretora de Enfermagem, observando-se a necessidade institucional. Essa proposta foi aprovada pela Direção Geral do Hospital.

A partir da ampliação da abrangência de atuação da Comissão, a então mestranda mobilizou alguns enfermeiros para formar um grupo de trabalho interno no referido hospital. Participaram desse grupo, além da mestranda, a Assessora da Diretoria de Enfermagem, outra mestranda que estava trabalhando a temática e uma enfermeira estomaterapeuta, mestre em Tecnologias em Saúde.

\section{Estruturação}

$\mathrm{Na}$ etapa de estruturação, o grupo de trabalho passou a ser denominado Núcleo Estruturador, que a partir de então passou a realizar reuniões periódicas quinzenais a fim de estruturar a Comissão a ser implantada. Essa fase aconteceu entre junho e outubro de 2012. Nela couberam as deliberações quanto à definição do nome da Comissão, à organização em subgrupos específicos, à definição dos componentes e ao planejamento de trabalho.

O nome da Comissão foi alterado de Comissão de Prevenção e Tratamento de Feridas para Comissão de Cuidados com a Pele (CCP). Optou-se por formar quatro subgrupos, cada um com a coordenação de uma das enfermeiras do núcleo estruturador, sendo eles: úlceras crônicas, feridas agudas e cirúrgicas, estomias e cateteres e drenos. Para cada subgrupo, foram convidadas mais três enfermeiras que atuavam na assistência nas áreas relacionadas. A reunião dos quatro subgrupos passou a ser chamado de "Grande Grupo da CCP”.

Foram definidas reuniões quinzenais dos subgrupos, nas quais deveria ser definida a lesão prioritária naquela área, para estudo e elaboração de estratégias de prevenção e/ou tratamento.

O Grande Grupo se reuniria uma vez por mês, com duração de duas horas. Nessa reunião, seria repassado o andamento do trabalho dos subgrupos e tomadas decisões que competiam a todos, e haveria uma aula ou palestra para capacitação do grupo. Cada encontro seria de responsabilidade de um subgrupo.

Ainda na fase de estruturação, o núcleo estruturador realizou um levantamento dos produtos, para prevenção e tratamento de feridas, já padronizados no hospital por outros serviços, solicitando aumento de cota daqueles que poderiam ser utilizados para as lesões de maior prevalência nos pacientes internados. 
Além de aumento de cota de produtos padronizados, foram chamados representantes e assessores técnicos das empresas que fabricam ou comercializam curativos especiais, que apresentaram ao núcleo estruturador toda a sua linha de produtos. Foram selecionados produtos com potencial de padronização no âmbito técnico e financeiro para serem utilizados e avaliados. Depois de um período de utilização, foram realizadas algumas solicitações de padronização.

Com o auxílio da equipe do setor de Faturamento e Informática, criou-se uma Unidade Executante para a CCP, inserida no Sistema de Informação Hospitalar, e elaborou-se uma lista de códigos compatíveis com os procedimentos que seriam realizados pelos membros $\mathrm{da}$ $\mathrm{CCP}$. Esses códigos seriam registrados para servir de base de dados para pesquisas, levantamento de produção e cobrança pelos procedimentos.

Procedimentos operacionais e o fluxograma padrão foram desenvolvidos e inseridos no software de gerenciamento de documentos, descrevendo os passos da primeira avaliação de um paciente pelos membros da Comissão, bem como seu acompanhamento. Tais procedimentos ficaram disponíveis para consulta por todos os serviços.

\section{Documentação}

A documentação está descrita como uma etapa, por motivos didáticos, porém ocorreu desde a fase de negociação e continua acontecendo após a fase de implantação, fazendo parte de um processo dinâmico.

O projeto de implantação da Comissão foi o primeiro documento elaborado, com base no pré-projeto apresentado à Diretoria de Enfermagem. Foi elaborado segundo normas institucionais, contendo objetivos, justificativa, resultados esperados e propostas de ações. Depois de elaborado pelo núcleo estruturador, foi apresentado aos membros da Comissão, em reunião para alterações e aprovação. O projeto foi aprovado pela Diretoria de Enfermagem e posteriormente pela Direção Geral, que, a partir da aprovação, emitiu a portaria de nomeação dos membros.

O regimento da Comissão também foi elaborado pelo núcleo estruturador. Tendo como modelo outros regimentos da instituição, regulamenta as finalidades, composição da $\mathrm{CCP}$ e atribuições de seus membros.

Ainda na documentação, foram preenchidos impressos de solicitação de padronização de materiais, realizados ofícios solicitando aumento de cotas e abertura de uma unidade executante no Sistema de Informação Hospitalar, registrados os procedimentos no sistema, gerados relatórios de produtividade, produzidos materiais para os meios de comunicação institucionais, entre outros.

\section{Implementação}

Considerou-se implementação o funcionamento da CCP. Como citado nas outras etapas, ele não aconteceu somente depois das fases anteriores, mas concomitantemente a elas.

Pode-se citar nesta fase a concretização dos cronogramas de reuniões do Grande Grupo, dos subgrupos e do núcleo estruturador e, concomitante a elas, os atendimentos realizados, a partir de pedidos de consulta, a pacientes com lesões ou em risco.

As reuniões do núcleo estruturador se mantiveram para determinação do andamento da $\mathrm{CCP}$, e as reuniões do Grande Grupo seguiram com apresentação das atividades dos subgrupos, com capacitações e discussões quanto ao andamento.

Os atendimentos foram iniciados de maneira não sistematizada, por meio de pedidos informais da equipe de Enfermagem e médica, para avaliação de pacientes com feridas complexas, que eram realizados pela enfermeira estomaterapeuta do núcleo estruturador. Na sequência, após desenvolvimento de fluxograma e procedimento operacional padrão, os atendimentos passaram a ser realizados por uma das enfermeiras do núcleo estruturador, por meio de pedidos de consulta, registrados no Sistema de Informação Hospitalar e evoluídos no prontuário do paciente. Além disso, as enfermeiras deixavam orientações por escrito afixadas próximo ao leito do paciente e orientavam a equipe de Enfermagem, o paciente e o acompanhante.

Durante um ano de funcionamento da CCP, os membros se dividiam entre suas atribuições nas unidades de lotação e as avaliações e os acompanhamentos de pacientes. Depois desse período, por necessidade institucional, observada pela demanda de atendimentos, foi aberta uma vaga para enfermeiro de dedicação exclusiva à CCP. Por meio de processo seletivo interno, a enfermeira estomaterapeuta do núcleo estruturador passou a ocupar essa vaga.

A partir da observação de demanda no primeiro ano de funcionamento da CCP, algumas modificações foram realizadas: os instrumentos de avaliação das lesões deixaram de ser utilizados, dando lugar à evolução sistematizada na 
ficha de evolução clínica do prontuário do cliente, a fim de fortalecer a Sistematização da Assistência de Enfermagem, também em fase de implementação.

Para fortalecimento do Grande Grupo, tendo em vista que as demandas de atendimento para cada subgrupo eram muito diferenciadas, cancelaram-se suas reuniões e aumentou-se a periodicidade de reuniões gerais, inserindo nelas as discussões de todas as áreas.

Entre as atividades relevantes da implementação da CCP, pode-se citar a seleção de Diagnósticos de Enfermagem da NANDA para problemas relacionados às lesões de pele e complicações periestomais, com suas respectivas prescrições de Enfermagem e inserção no Sistema de Informação Hospitalar.

Outra atividade importante foi a campanha denominada "HC livre de UP", que teve por objetivo a conscientização de toda a equipe de saúde quanto às medidas preventivas de úlceras por pressão, por meio de capacitação nas unidades de internação, com distribuição de materiais informativos, além de encontro científico com o tema "Úlceras por Pressão: Impacto e Prevenção” e auditorias.

A adoção de úlceras por pressão como prioridade de atuação preventiva da CCP baseia-se na prevalência delas, que pode ser observada pelos dados de atendimento descritos na sequência.

Nos últimos seis meses de atuação da CCP, a partir da lotação exclusiva de uma enfermeira na Comissão, foram solicitadas 184 avaliações por meio de pedidos de consulta. Grande parte dos pacientes avaliados apresentava mais de uma ferida e todos eles foram acompanhados semanalmente até a alta hospitalar, óbito, cicatrização completa da lesão ou redução de risco. Nesse período, 131 lesões avaliadas foram úlceras por pressão, em todos os estágios, com predominância para estágios III, IV e inclassificável.

\section{DISCUSSÃO}

Para a efetiva implantação da Comissão, foi de fundamental importância esta ter sido proposta como projeto de dissertação do mestrado profissional vinculado à mesma universidade que a instituição. $\mathrm{O}$ mestrado profissional tem como objetivo a intervenção da prática profissional, visando melhorar a eficácia e a eficiência das instituições por meio da solução de problemas, do desenvolvimento e da aplicação de processos de inovação apropriados à instituição ${ }^{8}$.
Vale ressaltar a linha e o grupo de pesquisa ao qual o tema da dissertação citada foi vinculado - Tecnologia e Inovação em Saúde (TIS) -, mesma área de mestrado de outra enfermeira do núcleo estruturador. Ocorreu assim a integração da academia com a prática, ou seja, universidade e unidade hospitalar, contribuindo para a melhoria do cuidado baseado nas melhores evidências .

A implantação de uma Comissão de Cuidados com a Pele relaciona-se com os três tipos de tecnologias definidas por Merhy como tecnologia dura, dura-leve e leve. Segundo o autor, tecnologia dura é todo tipo de equipamentos e medicamentos utilizados em intervenções terapêuticas, como coberturas especiais, bolsas coletoras e outros produtos. A tecnologia dura-leve é o olhar do profissional de saúde sobre o usuário, fundamentado em saberes bem definidos, como o conhecimento acerca das fases de cicatrização para guiar a definição da terapêutica adequada. A tecnologia leve é a que permite a produção de relações envolvidas no encontro trabalhador-usuário mediante a escuta, o interesse e a construção de vínculos que possibilita captar a singularidade ${ }^{10}$.

Segundo autores, as comissões devem ser um grupo técnico com caráter normativo e consultivo para desenvolver, organizar e coordenar atividades relacionadas à assistência, educação permanente e pesquisa no que se relaciona à prevenção e ao tratamento de lesões de pele ${ }^{11}$.

Considera-se imprescindível o envolvimento inicial da alta gestão da instituição, sem a qual não teriam sido possíveis os próximos passos dados em direção à aprovação dela nos colegiados institucionais.

A ampliação de abrangência de atuação de uma Comissão de Prevenção e Tratamento de Feridas para uma Comissão de Cuidados com a Pele possibilitou a cobertura de atendimento a pacientes com problemas referentes a estomas intestinais e urinários, assim como complicações em locais de inserção de drenos e cateteres de alimentação e eliminação, empregando assim o conhecimento e a atuação da enfermeira especialista em Estomaterapia que faz parte do núcleo estruturador.

Conforme descreve o estatuto da Associação Brasileira de Estomaterapia, o estomaterapeuta é um enfermeiro especialista, com conhecimento e prática voltada para a assistência de pessoas com estomias, fístulas, tubos, cateteres e drenos, feridas agudas e crônicas, e incontinências anal e urinária, em seus aspectos preventivos, terapêuticos e de reabilitação, em busca da melhoria da qualidade de vida ${ }^{12}$. 
Além do exposto, o nome da Comissão facilitou o entendimento dos profissionais da instituição para a abrangência de atuação dela, que passou a ser chamada não somente para atendimento de pacientes com feridas complexas mas também para avaliação de alterações como hiperemias e descamações, podendo atuar assim na prevenção de situações mais severas.

Autores ressaltam a importância da abordagem preventiva na assistência do enfermeiro, incentivando o uso de estratégias eficazes de prevenção como ferramenta no gerenciamento do cuidado, evitando que a assistência prestada resulte em dano ao paciente ${ }^{4}$.

Para garantir um atendimento de qualidade pela CCP, foi fundamental o engajamento de profissionais com experiência clínica e conhecimento científico em seu núcleo estruturador. Entre esses, destacam-se a idealizadora da Comissão, que desenvolveu sua dissertação com o objetivo de implantar a Comissão e que tem experiência de 18 anos na prevenção e no tratamento de feridas, tendo atuado na implantação, estruturação e assistência dos ambulatórios de Tratamento de Feridas e de Pé Diabético, e uma enfermeira com especialização em Estomaterapia.

Segundo pesquisadores, os profissionais escolhidos para compor a Comissão devem estar envolvidos e cientes de suas responsabilidades na prevenção e tratamento de lesões de pele, sendo necessário que pelo menos um dos componentes seja especialista na área ${ }^{13}$.

A organização de um núcleo estruturador, um número pequeno de membros engajados e capacitados que trabalharam na estruturação da Comissão antes de propor o funcionamento desta, foi válida para garantir a otimização dos tempos de reuniões e outras atividades realizadas para que a implantação fosse possível. Se todas as discussões tivessem sido propostas em reunião geral, com participação de todos os membros sugeridos, certamente o processo teria sido mais moroso, com divergência de ideias e sem o mesmo nível de comprometimento de todos os envolvidos, tendo em vista interesses pessoais, profissionais e demanda de trabalho.

O envolvimento dos profissionais do núcleo estruturador e a periodicidade frequente das reuniões iniciais favoreceram para que a negociação e a estruturação acontecessem em um período de quatro meses para início efetivo das atividades da Comissão.

A CCP foi inicialmente estruturada com a formação de subgrupos específicos. Com o andamento da Comissão, observou-se um enfraquecimento do "Grande Grupo" em decorrência da demanda científica de trabalho dos subgrupos. As reuniões dos subgrupos eram quinzenais, enquanto a geral era mensal. Houve faltas frequentes às reuniões gerais, as demandas dos subgrupos foram desiguais e os membros ficavam com conhecimento limitado à sua área. Por essas razões, a estrutura de subgrupos foi desfeita e as reuniões gerais passaram a ser quinzenais, com abordagem de temas referentes a todas as áreas, de acordo com a demanda institucional.

Fica a possibilidade de reestruturação em subgrupos para o momento em que houver uma Comissão fortalecida, com membros conhecedores de todas as suas áreas de abrangência e com clareza sobre a sua preferência de atuação para atender à demanda específica daquela área. É importante também maior divulgação na instituição sobre as áreas de abrangência da Comissão, para que a demanda seja estendida para todos os subgrupos.

Outra adequação percebida como necessária a partir do andamento da Comissão foi o instrumento de avaliação inicial e acompanhamento dos pacientes deixar de ser utilizado, dando lugar à evolução clínica sistematizada no prontuário do paciente em folha de evolução de uso multiprofissional.

Essa decisão foi tomada a fim de fortalecer a implantação da Sistematização da Assistência de Enfermagem na instituição, que padronizou a avaliação subjetiva e objetiva do paciente, os Diagnósticos de Enfermagem por áreas e prescrições relacionadas. Salientamos que essa alteração possibilitou maior visibilidade da Comissão por outros profissionais, uma vez que o registro de avaliação e acompanhamento das lesões passou a ser realizado no prontuário do paciente na folha de evolução de uso multiprofissional, que é uma ferramenta efetiva de comunicação entre as categorias.

De qualquer maneira, a elaboração dos instrumentos de avaliação, pela Comissão, foi uma fase importante de capacitação, para que todos os membros interiorizassem uma sequência clara de avaliação, passando essa a ser automática para a evolução sistêmica adotada.

No âmbito da capacitação, todas as discussões e explanações foram de fundamental importância para a formação dos membros, no sentido de estimular raciocínio clínico na área e padronizar condutas que embasarão a elaboração de protocolos institucionais.

Autores citam que a eficácia da prevenção e do tratamento de feridas depende do conhecimento dos profissionais quanto à fisiologia da pele, ao processo de cicatrização 
e à utilização de protocolos adequados e consistentes e da orientação e educação do paciente, familiar e/ou cuidador, tornando-os participantes ativos do processo $^{14}$.

Entre os assuntos necessários a serem inseridos em programas de capacitação de comissões que envolvem tratamento e prevenção de feridas, está o conhecimento de produtos disponíveis no mercado e suas particularidades. Faz-se necessário o conhecimento da tecnologia disponível para questionar seus benefícios e diferenciais, assim como ter embasamento científico para discussão de padronizações baseada na necessidade institucional.

Sabe-se da necessidade de realização de estudos comparativos entre os períodos anterior e posterior à implantação da Comissão para demonstrar a efetividade de tal medida no que diz respeito ao processo cicatricial das lesões crônicas, prevenção de feridas evitáveis e complicações de lesões intencionais (incisões cirúrgicas, estomas, drenos, entre outras). Até a elaboração do estudo, não houve tempo hábil para a realização de tais estudos em razão da demanda de estruturação, acompanhamento de pacientes e ações educativas, porém foi possível verificar, por meio de observação documentada de residentes de Enfermagem, um aumento no percentual de adesão dos profissionais às medidas de prevenção de úlceras por pressão.

O investimento financeiro por parte da instituição foi possível respeitando a burocracia processual e a limitação de recursos de um hospital público. Estudos de custo e efetividade foram necessários para demonstrar o benefício institucional com o encaminhamento dos processos de padronização.

\section{CONCLUSÃO}

Considera-se efetiva a estratégia utilizada para implantação de uma Comissão de Cuidados com a Pele descrita neste estudo. Sua efetividade pode ser observada atualmente pelo impacto positivo dela na instituição, pelo número crescente de pedidos de consultas por diversas categorias profissionais, inclusive para prevenção de lesões, pela evolução favorável das lesões, pelo uso adequado da tecnologia disponível e pelo envolvimento de profissionais de diversas áreas na campanha de prevenção de úlceras por pressão.

Pode-se destacar como ganho institucional a elaboração de protocolos que orientam condutas padronizadas, com o uso racional e adequado da tecnologia disponível, evitando gastos desnecessários e riscos aos pacientes. Além do citado, o enfoque em prevenção de lesões evitáveis e a utilização adequada de tecnologias estão em consonância com o movimento mundial de segurança do paciente.

Sugere-se que a formação de grupos específicos em uma Comissão seja discutida após solidificação de um grande grupo, com membros comprometidos que possam atuar em suas áreas de preferência. Reforça-se a importância de capacitação dos membros em todas as áreas da Comissão.

Como sugestão para estratégias similares, destaca-se a importância de participação de membros de outras categorias profissionais em Comissões de Cuidados com a Pele, como nutricionistas, fisioterapeutas, médicos, psicólogos, terapeutas ocupacionais e assistentes sociais, a fim de enriquecer as discussões de condutas e torná-las mais resolutivas.

\section{REFERÊNCIAS}

1. World Health Organization. World Alliance for Patient Safety, Taxonomy. The conceptual framework for the international classification for patient safety. Version 1.1. Final Technical Report. WHO: Geneva; 2009.

2. Lima LF, Leventhal LC, Fernandes MPP. Identificando os riscos do paciente hospitalizado. Einstein. 2008;6(4):434-8.

3. Oliveira RM, Leitão IMTA, Silva LMS, Figueiredo SV, Sampaio RC, Gondim MM. Estratégias para promover segurança do paciente: da identificação dos riscos às práticas baseadas em evidências. Esc Anna Nery. 2014;18(1): 122-9.

4. Domansky RC. Introdução. In: Domansky RC, Borges EL (orgs). Manual para prevenção de lesões de pele: recomendações baseadas em evidências. Rio de Janeiro: Rubio; 2012. p. 1-8.
5. Ferreira AM, Bogamil DDD, Tormena PCO. O enfermeiro e o tratamento de feridas: em busca da autonomia do cuidado. Arq Ciênc Saúde. 2008;15(3):105-9.

6. Salomé GM. Avaliando lesão: práticas e conhecimento dos enfermeiros que prestam assistência ao indivíduo com ferida. Rev Saúde Coletiva. 2009;6(35);280-7.

7. Hospital de Clínicas da Universidade Federal do Paraná [Internet]. Curitiba [cited 2012 Nov 13]. Available from: http://www.hc.ufpr.br/?q=node/65

8. Portaria Normativa/MEC $n^{0} 17$, de 28 de dezembro de 2009. Dispõe sobre o mestrado profissional no âmbito da Fundação Coordenação de Aperfeiçoamento de Pessoal de Nível Superior-CAPES. Brasília (DF): Diário Oficial da República Federativa do Brasil, 29 dez 2009: Seção 1: 20. 
9. Johann DA, Mingorance P, De Lazzari LSM, Pedrolo E, Almeida TQR, Danski MTR. Elaboração de diretriz clínica: integração entre universidade e unidade hospitalar. Cogitare Enferm. 2012;17(2):377-80.

10. Merhy EE. Saúde: a cartografia do trabalho vivo. $2^{a}$ ed. São Paulo: Hucitec; 2005.

11. Sodevilla-Agreda JJ, Garcia-Fernandes FP. Comisiones de Úlceras por Presión. Serie Documentos de Posicionamento GNEAUPP $n^{\circ}$ 1. (Segunda Edición). Grupo nacional para el Estudio y Asesoramiento em Úlcera por Presíon y Heridas Crónicas. Logroño. 2012.
12. Associação Brasileira de Estomaterapia: estomias, feridas e incontinências. Competências do Enfermeiro Estomaterapeuta (ET) ou do Enfermeiro Pós-graduado em Estomaterapia (PGET). Estima. 2008;6(1):33-43.

13. Declair V. Comissão de curativos estruturação de serviço de prevenção e tratamento de feridas em uma instituição hospitalar [Internet] [cited 2014 Fev 11]. Available from: http://www.vdeclair.com.br/doc/comissao_curativos

14. Carneiro CM, Sousa FB, Gama FN. Tratamento de feridas: assistência de enfermagem nas unidades de atenção primária à saúde. Rev Enfermagem Integrada. 2010;3(2):495-505. 